\title{
PSYCHOLOGICAL WELL-BEING AND INTERNALIZING DIFFICULTIES IN ITALIAN EMERGING ADULTS
}

In Happiness And Contemporary Society : Conference Proceedings Volume (Lviv, March, 20-21, 2021). Lviv: SPOLOM, 2021. P. 54-58. https://doi.org/10.31108/7.2021.9

ISBN 978-966-919-697-2 
https://doi.org/10.31108/7.2021.9

BURATTA Livia

Phd Candidate in Human Science,

Department of Philosophy, Social Science and Education, University of Perugia

(Perugia, Italy)

DELVECCHIO Elisa

PhD, Assistant Professor in Psychology

Department of Philosophy, Social Science and Education, University of Perugia

(Perugia, Italy)

MAZZESCHI Claudia

PhD, Professor in Psychology

Department of Philosophy, Social Science and Education, University of Perugia

(Perugia, Italy)

\section{PSYCHOLOGICAL WELL-BEING AND INTERNALIZING DIFFICULTIES IN ITALIAN EMERGING ADULTS}

The new theoretical view of well-being considers happiness not only as "feeling good" psychologically but, in a wider perspective, as an optimal experience in human functioning.

In the last years research has examined the trajectory of psychological well-being (PWB) over the lifespan, in particular during different life transition period, and its relationship with psychological health. Few studies specifically focused on emerging adulthood, a life stage characterized by many developmental challenges. As conceived by Ryff's model of PWB, it is a complex and multifaced construct with different dimensions.

The aims of this paper were to explore the PWB dimensions in a specific population of Italian emerging adults and to analyse the link between the psychological well-being and internalizing emotional difficulties.

Findings showed in Italian EA higher scores in specific dimensions of PWB such as personal growth and purpose in life, and lower scores in self-acceptance and environmental mastery. Moreover, results highlighted negative relationships between all PWB dimensions with internalizing difficulties.

This study suggests the importance of exploring the specificities of PWB both in clinical and health contexts in order to promote more tailored interventions specifically aimed for internalizing difficulties.

KEY WORDS: Psychological well-being, happiness, internalizing difficulties, emotional difficulties, emerging adulthood.

\section{INTRODUCTION}

Over the last four decades research in psychology focused attention on health and well-being, considering them not only as absence of illness or unhappiness, but as an optimum human functioning (Diener, 1984; Ryff \& Singer, 1996).

In psychology, well-being is considered a dimension of happiness. Researchers suggest that being happy does not necessarily mean being psychologically well, as highlighted by the well-being eudonic perspective (Ryff \& Singer, 2008). Indeed, in a eudemonic perspective people can be considered to be psychologically well and happy 
when are able "to develop their abilities and personal growth, considering the latter as the main indicators of positive functioning" (Ryan and Deci, 2001 p. 32). One of the most consolidated well-being eudemonic perspectives due to its theoretical foundations is the Ryff' model of psychological well-being. The author has brought together the humanistic psychological tradition and the concepts of self-realization, individuation, maturity and the full functioning of the person in a single theory.

Through a developmental model aimed to examine the trajectory of well-being over the lifespan, Ryff \& Keyes (1995) operationalized the psychological well-being (PWB) along six distinct dimensions: Autonomy, considered a sense of selfdetermination and independence of the person; Environmental Mastery, the person's ability to manage the environment and to effectively use the opportunities it provides; Personal Growth, the sense of growth and development as a person; Positive Relationships, having or developing quality relationships with others; Purpose in Life, the belief that for the person their life experience has a meaning and a purpose, and finally Self-Acceptance, considered the positive self-evaluation that the person has including past and present.

Many researchers have used the PWB model to investigate human development and psychological growth during lifespan. Cross-sectional and longitudinal studies from multiple large samples (Springer et al., 2011) showed some difficulties in specific aspects of well-being in different life moments. In fact, specific dimensions of eudaimonic well-being seem to improve or decline with age, in particular in specific moments of life transition (Mayordomo et al., 2016; Meléndez et al., 2018). These studies showed that autonomy, environmental mastery and self-acceptance reach higher scores with advancing age, while positive relationship with other, purpose in life and personal growth show higher score among younger people.

How individuals facing life transitions is related to the PWB perception. To date literature has focused particularly on transition from adulthood to aging, few studies are focused on other transition periods over the lifespan, such as adolescence and, even less so, emerging adulthood (EA, Arnett, 2004). EA is the period from adolescence to adulthood, considered the age of identity exploration and the age of possibilities in which the people change their lives as to work, residence and relationships. Due to all these life changes and developmental challenges, EA is also characterized by instability and then it is very sensitive time (Arnett, 2004). The life events characterized this developmental stage can seriously impact on mental health, in particular on affective and emotional dimensions (Germani et al., 2020).

In recent years Ryff's theoretical model of well-being has been studied in correlation with mental health. The research has shown its relevance for multiple aspects of mental functioning (Ryff, 2014).

Some study has shown that the PWB dimensions are often impaired in people with mood and anxiety disorders, more specifically, lack of PWB has been found to be a risk factor for emotional difficulties (Fava and Tomba, 2009). Similar results were found in specific EA samples (Rapee et al., 2015; Yang and Chang, 2016) but still few studies confirming this in Italian population (Inguglia et al., 2015).

Given the increased research interest in recent years about the study of PWB and its link with mental health, and the little research specifically focused on life transitional period of EA, the purposes of this cross-sectional study were to explore the levels of PWB, in all its dimension, in Italian EA. Subsequently, to analyse the link between the PWB dimensions and internalizing psychological difficulties in this specific population. 


\section{METHOD}

Participants and Procedures

Participants were 468 EA recruited from Italian Universities through a convenience sampling following these inclusion criteria: (1) agreed to participate after reading the study description, (2) age between 18 and 29 years and (3) completed all measures of the questionnaire. The $51.1 \%$ were males and $48.9 \%$ were females, the mean age was $21.90(\mathrm{Sd}=1.78 ; \mathrm{Min}=18 ; \mathrm{Max}=29)$. This study was conducted in line with Association Italian Psychology (AIP) Code of Conduct. The participation was voluntary, anonymous and no incentive reward was given. All participants could withdraw at any moment.

\section{Measures}

Psychological well-being scale (PWBS, Ryff \& Keyes, 1995). Self-report measure composed by 84 items with a 6-points Likert scale form 1 (strongly disagree) to 6 (strongly agree) that assess six dimensions of the well-being: autonomy, environmental mastery, personal growth, positive relations with others, purpose in life and selfacceptance. For each dimension higher score shows higher mastery in the specific area.

Strengths and Difficulties Questionnaire (SDQ, Goodman \& Goodman, 2009). Self-report measure composed by 25 items with a 3-points Likert scale from 0 (not true) to 2 (certainly true) that assess the dimension of internalizing difficulties (emotional problems and peer problems) and the dimension of externalizing difficulties (conduct problems and hyperactivity). In this study has been used specifically the internalizing difficulties dimension. Higher score shows higher difficulties.

Data Analysis

Descriptive statistics were performed to explore the PWB levels in the Italian EA. Repeated Measures Analysis were used to highlight differences among levels of the six PWB dimensions in EA. Post hoc comparisons with Bonferroni's method were carried out. Subsequently, Pearson's Correlations was performed to analyse the relationship between the PWB dimensions and internalizing difficulties. Correlation effect sizes were interpreted according to Cohen (1988) with coefficients of $0.10,0.30$ and 0.50 representing small, medium and strong effect sizes, respectively. In all analyses performed, significance requires $\mathrm{p}$ value $<0.05$. All analysis was run using SPSS, release 18 (SPSS Inc., Chicago).

\section{Results}

In table 1 are reported the mean scores of each PWBS dimensions. The Repeated Measures and the Post-Hoc Analyses highlighted differences among all dimensions ( $\mathrm{F}=$ 107.84; $\mathrm{p}$ <.001). Participants reported higher score in personal growth dimension and lower score in self-acceptance dimension than all other ones (Post-Hoc $=3>4>5>1>2>6$ ).

Table 1: Mean and Standard Deviation (Sd) of PWB dimensions

\begin{tabular}{lcc}
\hline PWB dimensions & Mean & Sd \\
\hline Autonomy (1) & 58.32 & .44 \\
Environmental mastery (2) & 56.48 & .47 \\
Personal growth (3) & 63.64 & .41 \\
Positive relationship with other (4) & 61.47 & .53 \\
Purpose in life (5) & 60.00 & .54 \\
Self-acceptance (6) & 54.55 & .53 \\
\hline
\end{tabular}


Person's correlations (table 2) showed close relationship between all PWB dimensions and internalizing difficulties. In particular, environmental mastery, positive relation with other, purpose in life and self-acceptance dimensions were negatively related to internalizing difficulties with strong effect size, while, the personal growth and autonomy dimensions were negatively related to internalizing difficulties with medium effect size.

Table 2: Person's correlations

\begin{tabular}{lc}
\hline PWB dimensions & $\begin{array}{c}\text { SDQ } \\
\text { Internalizing }\end{array}$ \\
\hline Autonomy (1) & $-.303^{* *}$ \\
Environmental mastery (2) & $-.571^{* *}$ \\
Personal growth (3) & $-.300^{* *}$ \\
Positive relationship with other (4) & $-.502 * *$ \\
Purpose in life (5) & $-.522^{* *}$ \\
Self-acceptance (6) & $-.578^{* *}$ \\
\hline
\end{tabular}

\section{DISCUSSION AND CONCLUSION}

With the growth of studies on happiness and psychological well-being considered as full development of human potential, the purposes of this paper were to explore the levels of PWB perception following the Ryff's theoretical model, and its link with the emotional difficulties, in a specific population of EA, life transitional stage still little studied.

These findings showed that Italian EA referred higher level on the specific dimension of personal growth than other one. According to previous literature the personal growth considered as intentional involvement in changing and developing as a person through an escalating change, seeking out opportunities, and individual's personal fulfilment, was one of three dimensions, along with positive relationships with other and purpose in life, which achieves higher scores in this specific stage of life. Probably these results because the EA, compared to adults and elderly, have a longer time frame in which to explore the opportunities offered by life and to discover and pursue personal interests and goals (Mayordomo et al., 2016; Meléndez et al., 2018).

In line with other studies, the dimensions showing lower scores in EA were selfacceptance and environmental mastery (Ryff \& Keyes, 1995; Lane et al., 2016; Meléndez et al., 2018). According to EA theory (Arnett, 2044) the instability and continuous changes characterizing this specific life transitions stage, may often correspond to adult expectations making EA feel ill prepared, resulting probably in diminished self-acceptance and perceptions of environmental mastery. These patterns of PWB profile over lifespan should be validated with longitudinal evidence.

As aforementioned, life events during this specific developmental stage could seriously impact on the general perception of one's mood with an effect on state of psychological health as well. Until now most of the studies carried out on this population focused on the link between emotional problems with specific dimensions of PWB (i.e. autonomy, positive relationship with other, purpose in life and personal growth) (Takebayashi et al., 2017; Rapee et al., 2015; Bronk, 2014; Yung and Chang, 2016). Findings of this study founded that lower levels in all dimensions of PWB during EA were related to higher levels of emotional difficulties, in particular, stronger correlations have been highlighted between internalizing difficulties and environmental mastery, 
positive relationship with other, purpose in life and self-acceptance. Some studies hypothesized that poor perception in PWB dimensions could be risk factors to develop internalizing difficulties as lower levels of mood status, emotional difficulties or depressive symptoms (Inguglia et al., 2015; Fava and Tomba, 2009). Longitudinal studies are needed to explore and to confirm this direction.

Present study may have important implications for psychological health and general well-being in EA. These results highlighted the importance of considering the PWB dimensions as resources of the people on which to work to make the EA able to overcome the life' challenges characterizing this specific life transitional period.

Could be important to apply the Ryff's PWB model both in assessment and treatment phases. In fact, having the people differing combinations of strengths and vulnerabilities one has to intervene with what is available, so it is appropriate to explore them in order to allow intervention to rapidly focus on patterns that need to change to have a positive impact on internalizing dimensions and consequently on mental health, both in clinical and health context.

\section{REFERENCES}

1. Arnett, J. J. (2004). Emerging adulthood: The winding road from the late teens through the twenties. New York, NY: Oxford University Press.

2. Diener, E., (1984). Subjective well-being. Psychol Bull, 95, 542-575.

3. Germani, A., Delvecchio E., Li, J.B., \& Mazzeschi, C. (2020). Protective factors for depressive symptoms in emerging adulthood. Scandinavian Journal of Psychology, 61, 237-242.

4. Fava GA, Tomba E. (2009) Increasing psycho- logical well-being and resilience by psycho- therapeutic methods. J Personality.

5. Inguglia, C., Ingoglia, S., Liga, F., Coco, A. L., \& Cricchio, M. G. L. (2015). Autonomy and relatedness in adolescence and emerging adulthood: relationships with parental support and psychological distress. J. Adult Dev. 22, 1-13

6. Meléndez, J. C., Agustí, A. I., Delhom, I., Reyes, M. F., \& Satorres, E. (2018). Bienestar subjetivo y psicológico: comparación de jóvenes y adultos mayores. Summa Psicol. UST $15,18-24$.

7. Rapee, R. M., Peters, L., Carpenter, L., \& Gaston, J. E. (2015). The Yin and Yang of support from significant others: Influence of general social support and partner support of avoidance in the context of treatment for social anxiety disorder. Behaviour Research and Therapy, 69, 40-47.

8. Ryan, R. M., \& Deci, E. L. (2001). On happiness and human potentials: a review of research on hedonic and eudaimonic well-being. Annu Rev Psychol, 52, 141-166.

9. Ryff, C. D. \& Keyes, C. L. M. (1995). The structure of psychological well-being revisited. Journal of Personality and Social Psychology, 69, 4, 719-727.

10. Yang, H. \& Chang, E. C. (2014). Examining the Structure, Reliability, and Validity of the Chinese Personal Growth Initiative Scale-II: Evidence for the Importance of Intentional Self-Change among Chinese. Journal of Personality Assessment, 96(5), 1-8. 